\title{
Irreducibility properties of Keller maps
}

\author{
Michiel de Bondt* \\ Department of Mathematics, Radboud University \\ Nijmegen, The Netherlands \\ E-mail: M.deBondt@math.ru.nl \\ Dan $\operatorname{Yan}^{\dagger}$ \\ Department of Mathematics and Computer Science, \\ Hunan Normal University, Changsha 410006, China \\ E-mail: yan-dan-hi@163.com
}

September 24, 2018

\begin{abstract}
Jẹdrzejewicz showed that a polynomial map over a field of characteristic zero is invertible, if and only if the corresponding endomorphism maps irreducible polynomials to irreducible polynomials. Furthermore, he showed that a polynomial map over a field of characteristic zero is a Keller map, if and only if the corresponding endomorphism maps irreducible polynomials to square-free polynomials. We show that the latter endomorphism maps other square-free polynomials to square-free polynomials as well.

In connection with the above classification of invertible polynomial maps and the Jacobian Conjecture, we study irreducible properties of several types of Keller maps, to each of which the Jacobian Conjecture can be reduced. Herewith, we generalize the result of Bakalarski, that the components of cubic homogeneous Keller maps with a symmetric Jacobian matrix (over $\mathbb{C}$ and hence any field of characteristic zero) are irreducible.

Furthermore, we show that the Jacobian Conjecture can even be reduced to any of these types with the extra condition that each affinely linear combination of the components of the polynomial map is irreducible. This is somewhat similar to reducing the planar Jacobian Conjecture to the so-called (planar) weak Jacobian Conjecture by Kaliman.
\end{abstract}

Keywords. Jacobian Conjecture, Keller map, irreducible, square-free, weak Jacobian Conjecture.

MSC 2010. 14R15; 14R10; 12D05.

\footnotetext{
* Supported by the Netherlands Organisation for Scientific Research (NWO).

${ }^{\dagger}$ Supported by the National Natural Science Foundation of China (Grant No.11371343)
} 


\section{Introduction}

Throughout this paper, we will write $x$ for the $n$ indeterminates $x_{1}, x_{2}, \ldots, x_{n}$, where $n \in \mathbb{N}$. In a similar manner, we will write $y$ for $y_{1}, y_{2}, \ldots, y_{n}$ and $z$ for $z_{1}, z_{2}, \ldots, z_{n}$. $K$ always denotes a field of characteristic zero and $\bar{K}$ is the algebraic closure of $K$. Let $F=\left(F_{1}, F_{2}, \ldots, F_{m}\right) \in K[x]^{m}$. Then $F$ corresponds to the polynomial map $K^{n} \ni v \mapsto F(v) \in K^{m}$. Write $\mathcal{J} F$ for the Jacobian of $F$ with respect to $x$, i.e.

$$
\mathcal{J} F:=\mathcal{J}_{x} F:=\left(\begin{array}{cccc}
\frac{\partial}{\partial x_{1}} F_{1} & \frac{\partial}{\partial x_{2}} F_{1} & \cdots & \frac{\partial}{\partial x_{n}} F_{1} \\
\frac{\partial}{\partial x_{1}} F_{2} & \frac{\partial}{\partial x_{2}} F_{2} & \cdots & \frac{\partial}{\partial x_{n}} F_{2} \\
\vdots & \vdots & & \vdots \\
\frac{\partial}{\partial x_{1}} F_{m} & \frac{\partial}{\partial x_{2}} F_{m} & \cdots & \frac{\partial}{\partial x_{n}} F_{m}
\end{array}\right)
$$

Let $M^{\mathrm{t}}$ denote the transpose of a matrix $M$. For a single polynomial $f \in K[x]$, write $\nabla f$ for the gradient of $f$ with respect to $x$, i.e.

$$
\nabla f:=\nabla_{x} f:=\left(\mathcal{J}_{x} f\right)^{\mathrm{t}}=\left(\begin{array}{c}
\frac{\partial}{\partial x_{1}} f \\
\frac{\partial}{\partial x_{2}} f \\
\vdots \\
\frac{\partial}{\partial x_{n}} f
\end{array}\right) .
$$

Additionally, write $\mathcal{H} f$ for the Hessian of $f$ with respect to $x$, i.e.

$$
\mathcal{H} f:=\mathcal{J}_{x}\left(\nabla_{x} f\right)=\left(\begin{array}{cccc}
\frac{\partial}{\partial x_{1}} \frac{\partial}{\partial x_{1}} f & \frac{\partial}{\partial x_{2}} \frac{\partial}{\partial x_{1}} f & \cdots & \frac{\partial}{\partial x_{n}} \frac{\partial}{\partial x_{1}} f \\
\frac{\partial}{\partial x_{1}} \frac{\partial}{\partial x_{2}} f & \frac{\partial}{\partial x_{2}} \frac{\partial}{\partial x_{2}} f & \cdots & \frac{\partial}{\partial x_{n}} \frac{\partial}{\partial x_{2}} f \\
\vdots & \vdots & \ddots & \vdots \\
\frac{\partial}{\partial x_{1}} \frac{\partial}{\partial x_{n}} f & \frac{\partial}{\partial x_{2}} \frac{\partial}{\partial x_{n}} f & \cdots & \frac{\partial}{\partial x_{n}} \frac{\partial}{\partial x_{n}} f
\end{array}\right)
$$

We say that a polynomial map $F$ is invertible if $F$ has a polynomial inverse. So an invertible polynomial map is bijective. The converse holds if $K=\bar{K}$ (see [vdE, Th. 4.2.1]), but not in general (see [vdE, (1.1.36)]). The well-known Jacobian Conjecture (JC for short), raised by O.H. Keller in 1939 in [Kel], states that a polynomial map $F: K^{n} \rightarrow K^{n}$ is invertible if its Jacobian determinant $\operatorname{det} \mathcal{J} F$ is a nonzero constant. From [vdE, Th. 4.2.1] for $K=\mathbb{C}$ and [vdE, Prop. 1.1.12], one deduces that it suffices to prove that $F$ is injective in the definition of JC.

This conjecture has been attacked by many people from various research fields and remains open even for $n=2$ ! (Of course, a positive answer is obvious for $n=1$.) See $[\mathrm{BCW}]$ and $[\mathrm{vdE}]$ and the references therein for a wonderful 70 -years history of this famous conjecture. The condition that $\operatorname{det} \mathcal{J} F \in K^{*}$ is called the Keller condition and polynomial maps that satisfy this condition are called Keller maps.

Among the vast interesting and valid results, one result obtained by S.S.S. Wang in [Wan] in 1980 is that the JC holds for all polynomial maps of degree 2 
in all dimensions. Another result is the reduction to degree 3, due to $\mathrm{H}$. Bass, E. Connell and D. Wright in [BCW] in 1982 and A. Yagzhev in [Jag] in 1980, which asserts that the $\mathrm{JC}$ is true if it holds for all polynomial maps $F=x+H$, such that $H$ is cubic homogeneous, i.e. each component $H_{i}$ of $H$ is either zero or a cubic form.

Upon this reduction to the cubic homogeneous case, there are two subsequent reductions, but they cannot be applied both. The first one is that additionally, $H_{i}$ is a third power of a linear form for each $i$, see [Dru1]. The second one, which requires that the imaginary unit $\mathrm{i} \in K$, is that $\mathcal{J} H$ or equivalently $\mathcal{J} F$ is symmetric, see [dBvdE2]. By a special case of Poincaré's lemma, this is the same as that $F=\nabla f$ and $H=\nabla h$ for certain polynomials $f, h \in K[x]$. If both $H_{i}$ is a (third) power of a linear form and $H_{i}=\frac{\partial}{\partial x_{i}} h$ for each $i$, then $F=x+H$ is tame with inverse $x-H$, see [dBvdE1] and [Dru2, Th. 3.4].

In [Bak1, Th. 3.7], S. Bakalarski proved the following interesting connection between invertible polynomial maps and irreducibility: a Keller map from $\mathbb{C}^{n}$ to $\mathbb{C}^{n}$ is invertible, if and only if the corresponding endomorphism maps irreducible polynomials to irreducible polynomials. K. Rusek improved this result by showing that the Keller condition is not necessary. This improved result was generalized to arbitrary fields of characteristic zero in [Jẹd, Th. 5.2] by P. Jẹdrzejewicz. In [Jẹd, Th. 5.1], Jẹdrzejewicz proved the following counterpart of this result: a polynomial map from $K^{n}$ to $K^{n}$ is a Keller map, if and only if the corresponding endomorphism maps irreducible polynomials to square-free polynomials. We shall show in the next section that for Keller maps, the corresponding endomorphism even maps all square-free polynomials to square-free polynomials.

In $[\mathrm{Kal}], \mathrm{S}$. Kaliman showed that in order to prove the JC in dimension $n=2$ for $K=\mathbb{C}$ (and hence for all $K$ by [vdE, Prop. 1.1.12]), one may assume that $F_{1}+c$ is irreducible for every $c \in K$. To prove the JC in dimension $n \geq 3$ for $K=\mathbb{C}$ one may even assume that $F_{i}+c$ is irreducible for every $i \leq n$ and $c \in K$. This was proved in [KS, Th. 3]. We shall show that for the JC for all $n$, one may even assume that every affinely linear combination of the components of $F$ is irreducible. Furthermore, we combine this reduction with several other reductions of the JC, including both reductions in the previous paragraph. See Theorems 3.1 and 3.2.

In [Bak2], Bakalarski proved that each component of $F$ is irreducible if $F=$ $x+H, \operatorname{det} \mathcal{J} F=1, H$ is cubic homogeneous and $\mathcal{J} H$ is symmetric. (Actually, Bakalarski proved his result only for $K=\mathbb{C}$, but using Lefschetz' principle, one may assume that $K \subseteq \mathbb{C}$, which gives the general case.) Notice that $F_{i}$ is the image of $x_{i}$ under the corresponding endomorphism of $F$. We will generalize this result in i) of Theorem 3.3, where we show that $F_{i}$ is irreducible if $\mathcal{J} F$ is symmetric, $\operatorname{det} \mathcal{J} F \in K^{*}$ and $F_{i}=l+h$ such that $h$ and $l$ are homogeneous and $\frac{\partial}{\partial x_{i}} l \in K^{*}$. Notice that, as opposed to the result of Bakalarski, the index variable $i$ is free instead of bound by an universal quantifier. So the conditions on $l$ and $h$ are for $F_{i}$ only, and not for the $F_{j}$ with $j \neq i$.

Additionally, we show in Corollary 4.4 that $F_{i}$ is irreducible if $\operatorname{det} \mathcal{J} F \in K^{*}$ and the set of degrees of monomials of $F_{i}$ is $\{0,1,3\}$. If we combine this result 
with the above-mentioned result of Theorem 3.3, we can conclude that $F_{i}+c$ is irreducible for all $c \in K$ if $\mathcal{J} F$ is symmetric, $\operatorname{det} \mathcal{J} F \in K^{*}$, and $F=l+h$ such that $h$ is cubic homogeneous, $\operatorname{deg} l=1$, and $\frac{\partial}{\partial x_{i}} l \in K^{*}$. The latter result can also be found in i) of Theorem 3.3.

As an end of this introduction, we summarize some results in connection with coordinates. A polynomial $f \in K[x]$ is a coordinate if there exists an invertible polynomial map $F \in K[x]^{n}$ such that $f=F_{1}$. After some partial results in [vdES] and [Jel1], Z. Jelonek proved in [Jel2] that a polynomial map over $K$ is invertible, if and only if the corresponding endomorphism maps coordinates to coordinates. The result of [vdES, Lm. 2.3] by H. Derksen is that a polynomial map over $\bar{K}$ is a Keller map, if and only if the corresponding endomorphism maps linear coordinates to polynomials with nowhere vanishing gradients (for instance coordinates).

Obviously, Derksen's result is still valid if we replace 'linear coordinates' by 'coordinates'. It is however not true in general that a polynomial map over $K$ is invertible, if and only if the corresponding endomorphism maps linear coordinates to coordinates, see [MYZ, Th. 2.1] (so Derksen's result is only valid if $K=\bar{K}$ ). But C. Cheng and A. van den Essen proved in [CvdE, Th. 1.1], that in the case $n=2$, it indeed suffices to show that the images of linear coordinates are coordinates. Furthermore, A. van den Essen and V. Shpilrain showed in [vdES, Th. 1.2] that Keller maps $F$ are invertible if $F_{1}$ is a coordinate and the JC holds in dimension $n-1$.

\section{Some properties of Keller maps}

We start with a generalization of [Jẹd, Th. 4.1] by Jẹdrzejewicz. To be precise, [Jẹd, Th. 4.1] is the equivalence of 1) and 2) in the theorem below, for the case that $g$ is irreducible.

Theorem 2.1. Let $F \in K[x]^{n}$ be an arbitrary polynomial map. If $g \in K[x]$ is square-free, then the following conditions are equivalent:

1) $g \mid \operatorname{det} \mathcal{J} F$,

2) for every irreducible $\tilde{g} \mid g$, there exists an irreducible polynomial $\tilde{w} \in K[y]$ such that $\tilde{g}^{2} \mid \tilde{w}(F)$,

3) $g^{2} \mid w(F)$ for some square-free polynomial $w \in K[y]$.

Proof. Assume that $g \in K[x]$ is square-free. The equivalence of 1) and 2) follows by applying [Jẹd, Th. 4.1] for all irreducible polynomials $\tilde{g} \mid g$. To prove 2$) \Longrightarrow$ $3)$, take for $w$ in 3 ) the least common multiple of all $\tilde{w}$ appearing in 2). Then $w(F)$ in 3$)$ is a common multiple of the $\tilde{w}(F)$ appearing in 2). Since $g^{2}$ in 3) is the least common multiple of the $\tilde{g}^{2}$ appearing in 2), 2) $\Longrightarrow 3$ ) follows. Hence it remains to show 3$) \Longrightarrow 2$ ).

So assume 3 ) and let $\tilde{g}$ be an arbitrary irreducible divisor of $g$. We have to show that there exists an irreducible $\tilde{w} \in K[y]$ such that $\tilde{g}^{2} \mid \tilde{w}(F)$. Since 
$g^{2} \mid w(F)$, we can decompose $w=w_{1} w_{2}$, such that $w_{1}$ is irreducible and $\tilde{g} \mid$ $w_{1}(F)$. If $\tilde{g}^{2} \mid w_{1}(F)$, then we are done, so suppose that $\tilde{g}^{2} \nmid w_{1}(F)$. Then $\tilde{g} \mid w_{2}(F)$. Let $\bar{F}$ be the residue classes of $F$ modulo $\tilde{g}$, i.e. $\bar{F}_{i}=F_{i}+(\tilde{g})$ for each $i$. Define $r:=\operatorname{trdeg}_{K} K\left(\bar{F}_{1}, \bar{F}_{2}, \ldots, \bar{F}_{n}\right)$ and assume without loss of generality that $\bar{F}_{1}, \bar{F}_{2}, \ldots, \bar{F}_{r}$ are algebraically independent over $K$. Then $r \leq n-1$, because $w_{1}(\bar{F})=0$.

If $r \leq n-2$, then we can follow the last paragraph in the proof of (i) $\Longrightarrow$ (ii) of [Jẹd, Th. 4.1] verbatim to obtain that $\tilde{g}^{2} \mid \tilde{w}(F)$ for some irreducible $\tilde{w} \in K[y]$. So assume that $r=n-1$. Notice that $w_{1}$ and $w_{2}$ are relatively prime, because $w$ is square-free. Hence the ideal $\left(w_{1}, w_{2}\right)$ is not contained in a principal prime ideal of $K[y]$. Since $K[y]$ is a unique factorization domain, we can deduce that the ideal $\left(w_{1}, w_{2}\right)$ has height at least two. On the other hand, the ideal in $K[y]$ of algebraic relations between $\bar{F}_{1}, \bar{F}_{2}, \ldots, \bar{F}_{n}$ has height $n-r$ and contains $\left(w_{1}, w_{2}\right)$. So $n-r \geq 2$, which contradicts $r=n-1$.

One can also obtain a contradiction to $r=n-1$ by showing that the resultant with respect to $y_{n}$ of $w_{1}$ and $w_{2}$ is a nontrivial algebraic relation between $\bar{F}_{1}, \bar{F}_{2}, \ldots, \bar{F}_{n-1}$.

Just like [Jẹd, Th. 4.1], its immediate consequence [Jęd, Cor. 4.2] can be generalized. We do this by extending it with one line, namely property 3 ).

Corollary 2.2. Let $F \in K[x]^{n}$ be an arbitrary polynomial map. Then the following conditions are equivalent:

1) $\operatorname{det} \mathcal{J} F \in K^{*}$,

2) for every irreducible polynomial $w \in K[y]$, the polynomial $w(F)$ is squarefree,

3) for every square-free polynomial $w \in K[y]$, the polynomial $w(F)$ is squarefree.

Proof. Every assertion is equivalent to the nonexistence of an irreducible $g$ in the respective assertion of Theorem 2.1.

We end this section with a theorem about some reducibility properties which cannot be combined with the Keller condition. We use a result of [LM] for that.

Theorem 2.3. Let $F \in K[x]^{n}$ be a Keller map. Suppose that for each $i, F_{i}$ is of the form $L_{i} H_{i}$, where $\operatorname{deg} L_{i}=1$. Then the following statements are equivalent.

1) The linear part of $L_{i}(0) H_{i}$ is divisible by $L_{i}-L_{i}(0)$ for each $i$,

2) $L(0)$ is contained in the column space of $\mathcal{J} L$,

3) $\operatorname{det} \mathcal{J} L \in K^{*}$,

4) $\operatorname{deg} F=1$,

5) $F_{i}$ is irreducible for each $i$. 
Proof. Notice that 3$) \Longrightarrow 2$ ) and 4) $\Longleftrightarrow 5$ ) are trivial. Hence it suffices to prove the following.

1) $\Rightarrow 3)$ Suppose that 1) holds. Since $F_{i}=L_{i}(0) H_{i}+\left(L_{i}-L_{i}(0)\right) H_{i}$ for all $i$, we can deduce that for each $i$, the linear part of $F_{i}$ is equal to $\left(L_{i}-L_{i}(0)\right) c_{i}$ for some $c_{i} \in K$. Hence $c_{1} c_{2} \cdots c_{n} \operatorname{det} \mathcal{J} L=\left.(\operatorname{det} \mathcal{J} F)\right|_{x=0}$. Now 3$)$ follows from the Keller condition on $F$.

2) $\Rightarrow 3$ ) Let $*$ denote the Hadamard product and suppose that 2) holds. Say that $\mathcal{J} L \cdot a=L(0)$, where $a \in K^{n}$. Then the constant part of $L(x-a)$ is equal to

$$
L(-a)=\left.\mathcal{J} L\right|_{x=-a} \cdot(-a)+L(0)=\mathcal{J} L \cdot(-a)+\mathcal{J} L \cdot a=0 .
$$

Hence the linear part of $F(x-a)=L(x-a) * H(x-a)$ is equal to $L(x-a) *$ $H(-a)$. Using the Keller condition for $F, \operatorname{det} \mathcal{J} L|\operatorname{det}(\mathcal{J} F)|_{x=x-a} \in K^{*}$ follows, which is 3 ).

3) $\Rightarrow 4$ ) Suppose that 3) holds. Then $L$ is invertible and

$$
F\left(L^{-1}(x)\right)=L\left(L^{-1}(x)\right) * H\left(L^{-1}(x)\right)=x * H\left(L^{-1}(x)\right)
$$

is a Keller map as well. It follows from [LM, Prop. 6] that $\operatorname{deg} H=$ $\operatorname{deg} H\left(L^{-1}(x)\right)=0$. Hence $\operatorname{deg} F=1$.

4) $\Rightarrow$ 1) Suppose that $\operatorname{deg} F=1$. Then $\operatorname{deg} H_{i}=0$ and hence $L_{i}(0) H_{i} \in K$ for each $i$. Thus for each $i$, the linear part of $L_{i}(0) H_{i}$ is zero, which is divisible by $L_{i}-L_{i}(0)$.

\section{Irreducibility results for reductions of the JC}

Theorem 3.1. Assume $F \in K[x]^{n}$ is a cubic Keller map without quadratic part. Then there exists a $\lambda \in K^{n}$ such that for

$$
\begin{aligned}
& G=\left(F-\lambda x_{n+1}^{3}, x_{n+1}\right), \\
& G=\left(F-\lambda x_{n+1}^{3}, x_{n+1}, x_{n+2}+x_{n+1}^{3}\right),
\end{aligned}
$$

and

$$
\begin{aligned}
G & =\left(F-\lambda x_{n+1}^{3}, x_{n+2}-3 x^{\mathrm{t}} \lambda x_{n+1}^{2}, x_{n+1}\right) \\
& =(F, 0,0)+\nabla_{x, x_{n+1}, x_{n+2}}\left(x_{n+1} x_{n+2}-x^{\mathrm{t}} \lambda x_{n+1}^{3}\right),
\end{aligned}
$$

every linear combination of the components of $G$ and 1 which is reducible is already a linear combination of 1 .

Furthermore, $G$ is a cubic Keller map without quadratic part, and $F$ is invertible, if and only if $G$ is invertible. Additionally, we have the following.

i) If $F$ is linearly conjugate to a Druzkkowski map, then so is $G$ in (2). 
ii) If $\mathcal{J} F$ is symmetric, then so is $\mathcal{J}_{x, x_{n+1}, x_{n+2}} G$ in (3).

Proof. In Corollary 6.2, we will prove the first claim (the existence of $\lambda$ ) for

$$
G=\left(F-\lambda x_{n+1}^{3}, x_{n+1}, x_{n+2}-h\right),
$$

where $h \in K\left[x, x_{n+1}\right]$ is arbitrary. This immediately gives the first claim for $G$ in (2). To obtain the first claim for $G$ in (1) and (3), we remove the last component of $G$ and interchange the last two components of $G$ respectively in (4). Thus a $\lambda \in K^{n}$ as given exists.

By expansion of the determinant along the $(n+2)$-th column, if present, and subsequently along the last row, we see that

$$
\begin{aligned}
\operatorname{det} \mathcal{J}_{x, x_{n+1}} G & =\operatorname{det} \mathcal{J} F \text { in }(1), \\
\operatorname{det} \mathcal{J}_{x, x_{n+1}, x_{n+2}} G & =\operatorname{det} \mathcal{J} F \text { in }(2), \text { and } \\
\operatorname{det} \mathcal{J}_{x, x_{n+1}, x_{n+2}} G & =-\operatorname{det} \mathcal{J} F \text { in }(3) .
\end{aligned}
$$

Hence $G$ is a Keller map, and one can easily verify that $G$ is a cubic Keller map without quadratic part.

We only prove the rest of this theorem for the cases (2) and (3), since the case (1) is similar. Let $E=\left(x-\lambda x_{n+1}^{3}, x_{n+1}, x_{n+2}\right)$.

i) Assume that $G$ is as in (2). Then

$$
G=\left.E\left(F, x_{n+1}, x_{n+2}\right)\right|_{x_{n+2}=x_{n+2}+x_{n+1}^{3}} .
$$

Consequently, $F$ is invertible, if and only if $G$ is invertible.

Suppose that $T F\left(T^{-1} x\right)$ is a Drużkowski map. Set

$$
\tilde{T}=\left(\begin{array}{ccc|cc} 
& & & 0 & \\
& T & & \vdots & T \lambda \\
& & & 0 & \\
\hline 0 & \cdots & 0 & 1 & 0 \\
0 & \cdots & 0 & 0 & 1
\end{array}\right) .
$$

Then $\tilde{T} G\left(\tilde{T}^{-1}\left(x, x_{n+1}, x_{n+2}\right)\right)$ is a Drużkowski map as well. Hence $G$ is linearly conjugate to a Drużkowski map if $F$ is.

ii) Assume that $G$ is as in (3). Then

$$
\left(G_{1}, G_{2}, \ldots, G_{n}, G_{n+2}, G_{n+1}\right)=\left.E\left(F, x_{n+1}, x_{n+2}\right)\right|_{x_{n+2}=x_{n+2}-3 x^{\mathrm{t}} \lambda x_{n+1}^{2}} .
$$

Consequently, $F$ is invertible, if and only if $G$ is invertible.

Suppose that $\mathcal{J} F$ is symmetric. Then by (3), $\mathcal{J} G$ is symmetric as well, which completes the proof. 
Theorem 3.2. Assume that $F \in K[x]^{n}$ is a Keller map and let $d \geq 2$ be an integer. Then for

$$
\begin{aligned}
& G=\left(F-y^{* d}, y\right), \\
& G=\left(F-y^{* d}, y, z+y^{* d}\right),
\end{aligned}
$$

and

$$
\begin{aligned}
G & =\left(F-y^{* d}, z-d x^{\mathrm{t}} y^{*(d-1)}, y\right) \\
& =(F, 0, \ldots, 0,0, \ldots, 0)+\nabla_{x, y, z}\left(y^{\mathrm{t}} z-x^{\mathrm{t}} y^{* d}\right),
\end{aligned}
$$

every linear combination of the components of $G$ and 1 which is reducible is already a linear combination of 1 .

Furthermore, $G$ is a Keller map and $F$ is invertible, if and only if $G$ is invertible, and we have the following.

i) If $F-x$ is linearly conjugate to a power linear map of degree $d$, then so is $G-(x, y, z)$ in $(6)$.

ii) If $\mathcal{J} F$ is symmetric, then so is $\mathcal{J}_{x, y, z} G$ in (7).

Proof. In Corollary 5.5, we will prove the first claim for

$$
G=\left(F-y^{* d}, y, z-H\right),
$$

where $H \in K[x, y]^{n}$ is arbitrary. The proof of this theorem is a multidimensional variation of the proof of Theorem 3.1, where Corollary 5.5 is used by way of (8) instead of Corollary 6.2 by way of (4).

i) of Theorem 3.3 below is a generalization of [Bak2, Th. 2.2] by Bakalarski. In ii) of Theorem 3.3, (9) with $u=\mathrm{i}$ and $u^{\prime}=-\mathrm{i}$ corresponds to the gradient reduction of the JC in [dBvdE2]. Indeed, if $F=x+H, f=-x^{\mathrm{t}} \cdot H(x)$, and $f_{H}$ is as in Equation (3) of [dBvdE2] (i.e. $\left.f_{H}=-i y^{\mathrm{t}} \cdot H(x+\mathrm{i} y)\right)$, then

$$
\begin{aligned}
\frac{1}{2} \sum_{i=1}^{n} x_{i}^{2} & +\frac{1}{2} \sum_{i=1}^{n} y_{i}^{2}+f_{H} \\
& =\frac{1}{2}(x-\mathrm{i} y)^{\mathrm{t}} \cdot(x+\mathrm{i} y)-i y^{\mathrm{t}} \cdot H(x+\mathrm{i} y) \\
& =\frac{1}{2}(x-\mathrm{i} y)^{\mathrm{t}} \cdot((x+\mathrm{i} y)+H(x+\mathrm{i} y))-\frac{1}{2}(x+\mathrm{i} y)^{\mathrm{t}} \cdot H(x+\mathrm{i} y) \\
& =\frac{1}{2}(x-\mathrm{i} y) F(x+\mathrm{i} y)+\frac{1}{2} f(x+\mathrm{i} y),
\end{aligned}
$$

of which the gradient map is $\frac{1}{2} G$, where $G$ is as in (9) with $u=\mathrm{i}$ and $u^{\prime}=-\mathrm{i}$.

Taking $u=1$ and $u^{\prime}=-1$ in (9) gives a gradient reduction of the JC that does not require imaginary units, and $-\frac{1}{2} G$ has linear part $(-x, y)$ in that case, provided $F$ has linear part $x$ and $f$ has a trivial quadratic part. This linear 
part is also the result of the gradient reduction of the JC in [Dru2, Th. 3.1 (i)] (for cubic homogeneous maps). The reduction in [Dru2, Th. 3.1 (i)] is however slightly different, because it still requires a certain square root in $K$, namely $\sqrt{2}$.

Theorem 3.3. Assume that $G$ is a Keller map with a symmetric Jacobian over $K$. Write $G_{i}^{(1)}$ for the linear part of $G_{i}$.

i) If $G \in K[x]^{n}$, then $G_{i}(+c)$ is irreducible (for all $c \in K$ ) if $\frac{\partial}{\partial x_{i}} G_{i}^{(1)} \neq 0$ and $G_{i}-G_{i}^{(1)}$ is (cubic) homogeneous.

ii) If $G \in K[x, y]^{2 n}$, then $G_{i}$ is irreducible for all $i$ if $G$ is of the form

$$
G=\nabla_{x, y}\left(f(x+u y)+\left(x+u^{\prime} y\right)^{\mathrm{t}} F(x+u y)\right),
$$

where $u, u^{\prime} \in K$ such that $u \neq 0, f \in K[x]$ and $F_{i} \in K[x]$ for all $i \leq n$. Furthermore, $u \neq u^{\prime}$ and $F$ is a Keller map in this case, and additionally $F$ is invertible, if and only if $G$ is.

Proof.

i) Suppose that $G \in K[x]^{n}$ and $c \in K$ such that $G_{i}+c$ is reducible, $\frac{\partial}{\partial x_{i}} G_{i}^{(1)} \neq$ 0 and $G_{i}-G_{i}^{(1)}$ is homogeneous of degree $d$. Then $d \geq 2$. In order to prove i) both with and without the parenthesized parts, it suffices to obtain a contradiction in the case where either $d \leq 3$ or $c=0$. We shall derive a contradiction by showing that $\operatorname{deg} G_{i}=1$ in both subcases.

Since $G$ is a Keller map, we see by expansion of the Jacobian determinant of $G$ along the $i$-th row that $\mathcal{J} G_{i}$ is unimodular. In 1) $\Longrightarrow 3$ ) of Corollary 4.4, we will show that $\left(G_{i}^{(1)}\right)^{2} \mid G_{i}-G_{i}^{(1)}$ if $G_{i}-G_{i}^{(1)}$ is homogeneous of degree $d \geq 2$ and $\mathcal{J} G_{i}$ is unimodular, and either $d \leq 3$ or $c=0$. In Corollary 7.2 , we will show that $\operatorname{deg} G_{i}=1$ if $\frac{\partial}{\partial x_{i}} G_{i}^{(1)} \neq 0,\left(G_{i}^{(1)}\right)^{2}$ $G_{i}-G_{i}^{(1)}$ and $G$ is a Keller map with a symmetric Jacobian. Hence $\operatorname{deg} G_{i}=1$.

ii) Suppose that $G \in K[x, y]^{2 n}$ is as in (9). We can rewrite (9) as $G=$ $\nabla_{x, y}\left(\left.\left(f+y^{\mathrm{t}} F\right)\right|_{(x, y)=\left(x+u y, x+u^{\prime} y\right)}\right)$. Hence the chain rule for $\nabla_{x, y}=\mathcal{J}_{x, y}^{\mathrm{t}}$ tells us that

$$
G=\left.\left(\begin{array}{cc}
I_{n} & I_{n} \\
u I_{n} & u^{\prime} I_{n}
\end{array}\right)\left(\nabla_{x, y}\left(f+y^{\mathrm{t}} F\right)\right)\right|_{(x, y)=\left(x+u y, x+u^{\prime} y\right)} .
$$

In Lemma 7.5, we will show that $F$ is invertible, if and only if $\left(\nabla_{x, y}(f+\right.$ $\left.\left.y^{\mathrm{t}} F\right)\right)$ is, and that $\operatorname{det} \mathcal{H}_{x, y}\left(f+y^{\mathrm{t}} F\right)=(-1)^{n}(\operatorname{det} \mathcal{J} F)^{2}$. Since $G$ is a Keller map, we see that $u \neq u^{\prime}$ and that $F$ is a Keller map.

Again in Lemma 7.5, we will show that $\mu^{\mathrm{t}} \nabla_{x, y}\left(f+y^{\mathrm{t}} F\right)$ is irreducible for all $\mu \in K^{2 n}$ such that $\mu_{i} \neq 0$ for some $i \leq n$, provided $F$ is a Keller map. Thus $G_{i}$ is irreducible for all $i \leq n$. By assumption of $u \neq 0, G_{i}$ is irreducible for all $i>n$ as well. 
We end this section by showing that for components $F_{i}$ of power linear Keller maps over $K, F_{i}-c$ is irreducible for all $c \in K$.

Proposition 3.4. Assume that $f \in K[x]$, such that $\operatorname{deg} f \neq 1$ and $h:=f-x_{i}$ is a polynomial in a linear form. If $h \in K\left[x_{i}\right]$, then $f$ is not a component of a Keller map. If $h \notin K\left[x_{i}\right]$, then $f$ is a tame coordinate.

Proof. If $h \in K\left[x_{i}\right]$, then $f$ cannot be a component of a Keller map because $\mathcal{J} f$ is not unimodular. So assume $h \notin K\left[x_{i}\right]$. Then there exists a $T \in \mathrm{GL}_{n}(K)$ such that $T_{1} x=x_{i}$ and $h \in K\left[T_{2} x\right]$, say that $h=p\left(T_{2} x\right)$. It follows that $f$ is the first component of the composition of the elementary invertible map $\left(x_{1}+p\left(x_{2}\right), x_{2}, x_{3}, \ldots, x_{n}\right)$ and $T$.

Notice that i) of Theorem 3.3 and Proposition 3.4 are results about Keller maps of some type, rather than subsequent reductions to obtain irreducibility results. More results of this type are Theorem 5.1 and its corollary, and Theorems 6.1 and 7.3.

\section{Irreducibility lemmas for polynomials with uni- modular gradients}

Lemma 4.1. Let $f \in \bar{K}[x]$ such that $\operatorname{deg} f=d \geq 2$. Suppose that $f$ has monomials of degrees $0,1, d$ only, and that $x_{1}-c \mid f$ for some $c \in \bar{K}^{*}$. If $f$ is nonsingular, then

$$
f=c^{\prime}\left(x_{1}^{d}-c^{d}\right)+\left(c^{\prime \prime}-c^{\prime} d c^{d-1}\right)\left(x_{1}-c\right)
$$

for some $c^{\prime}, c^{\prime \prime} \in \bar{K}^{*}$.

Proof. Since $f$ is nonsingular, we have $f=\left(g \cdot\left(x_{1}-c\right)+c^{\prime \prime}\right) \cdot\left(x_{1}-c\right)$ for some $g \in \bar{K}[x]$ and $c^{\prime \prime} \in \bar{K}^{*}$. Hence

$$
h:=g \cdot\left(x_{1}-c\right)^{2}=f-c^{\prime \prime}\left(x_{1}-c\right)
$$

has monomials of degrees $0,1, d$ only. Let $h^{\prime}$ be the derivative of $h$ with respect to $x_{1}$, and take $c^{\prime} \in \bar{K}$ such that the constant part of $h^{\prime}$ is equal to $-c^{\prime} d c^{d-1}$ (if $h^{\prime}$ has one, otherwise take $c^{\prime}=0$ ). Since $h^{\prime}$ has only monomials of degree 0 and $d-1$, we deduce from $x_{1}-c \mid h^{\prime}$ that $h^{\prime}$ is completely determined by its constant part. More precisely,

$$
h^{\prime}=c^{\prime} d\left(x_{1}^{d-1}-c^{d-1}\right), \quad \text { so } \quad h=c^{\prime} x_{1}^{d}-c^{\prime} d c^{d-1} x_{1}+\left.h\right|_{x_{1}=0} .
$$

Since $x_{1}-c \mid h$ as well, it follows that $\left.h\right|_{x_{1}=0}$ is completely determined by the other monomials of $h$, i.e. the monomials of $h$ whose degree with respect to $x_{1}$ is positive. More precisely,

$$
h=c^{\prime}\left(x_{1}^{d}-c^{d}\right)-c^{\prime} d c^{d-1}\left(x_{1}-c\right) .
$$

By definition of $h, f$ is as claimed, where $c^{\prime} \neq 0$ because $\operatorname{deg} f \geq 2$. 
From now on in this section, we shall write $f^{(k)}$ and $g^{(k)}$ for the homogeneous part of degree $k$ of $f$ and $g$ respectively, or zero if $f$ or $g$ respectively has no such part.

Lemma 4.2. Let $f \in K[x]$ be nonzero. Assume that $f=g h$ is a polynomial decomposition, such that $h(0) \neq 0$. Take $g^{*} \in K[x]$.

If $g^{*} \mid f^{(0)}, f^{(1)}, \ldots, f^{(\operatorname{deg} g)}$, then $g^{*} \mid g$.

Proof. Notice that $g^{*}\left|f^{(0)}=g^{(0)} h(0)\right| g^{(0)}$. Suppose that $g^{*} \mid g^{(0)}, g^{(1)}, \ldots$, $g^{(i)}$ for some $i<\operatorname{deg} g$. Since $g^{*} \mid f^{(i+1)}$, we obtain by expressing $f^{(i+1)}$ in the homogeneous parts of $g$ and $h$ that $g^{*}\left|g^{(i+1)} h(0)\right| g^{(i+1)}$. By induction on $i$, $g^{*} \mid g^{(0)}, g^{(1)}, \ldots, g^{(\operatorname{deg} g)}$, so $g^{*} \mid g^{(0)}+g^{(1)}+\cdots+g^{(\operatorname{deg} g)}=g$.

Corollary 4.3. Assume $f \in K[x]$ such that $f-a x_{1}$ is homogeneous of degree $d \geq 2$ for some nonzero $a \in K$. If $f$ is reducible, then $x_{1} \mid f$.

Proof. Suppose that $f$ is reducible. Then we can decompose $f=g h$ such that $h(0) \neq 0$ and $\operatorname{deg} g \leq d-1$. From Lemma 4.2 with $g^{*}=x_{1}$, we obtain that $x_{1}|g| f$.

Corollary 4.4. Assume that $f \in K[x]$ has monomials of degree $0,1, d$ only, where $d \geq 2$, say that $f=f^{(0)}+f^{(1)}+f^{(d)}$. Suppose that $f$ is reducible and $\mathcal{J} f$ is unimodular. Then for

1) $d \leq 3$ or $f^{(0)}=0$,

2) $f$ has a divisor of degree 1 ,

3) $f^{(0)}=0, f^{(1)} \mid f$ and $\left(f^{(1)}\right)^{2} \mid f^{(d)}$,

we have 1) $\Longrightarrow$ 2) $\Longrightarrow$ 3).

Proof. Since $\mathcal{J} f$ is unimodular, we have $f^{(1)} \neq 0$ and $f$ is nonsingular over $\bar{K}$. Furthermore, $\operatorname{deg} f \geq 2$ because $f$ is reducible.

Since $2 \leq \operatorname{deg} f \leq d$, the case $d \leq 3$ of 1$) \Longrightarrow 2$ ) follows directly from the supposition that $f$ is reducible and the case $f^{(0)}=0$ of 1$) \Longrightarrow 2$ ) follows from Corollary 4.3 , because we may assume without loss of generality that $f^{(1)}=x_{1}$.

In order to prove 2$) \Longrightarrow 3$ ), suppose that $f$ has a divisor of degree 1 . Without loss of generality, we may assume that $x_{1}-c \mid f$ for some $c \in K$. From $\operatorname{deg} f \geq 2$, we can subsequently deduce that $\left(\frac{\partial}{\partial x_{1}} f 0 \cdots 0\right)$ is not unimodular. If $c \neq 0$, then Lemma 4.1 tells us that $\mathcal{J} f=\left(\frac{\partial}{\partial x_{1}} f \quad 0 \cdots 0\right)$ indeed, which contradicts that $\mathcal{J} f$ is unimodular. So $c=0$ and $x_{1} \mid f$. By the nonsingularity of $f$ over $\bar{K}$, we obtain that $f=c^{\prime} x_{1}\left(g x_{1}+1\right)$ for some $c^{\prime} \in K^{*}$ and $g \in K[x]$. This gives $3)$.

Lemma 4.5. Assume that $f \in \bar{K}[x]$ has degree at most $d$, and $g \in \bar{K}[y] \backslash \bar{K}$. Then for

1) $f-g$ is reducible, 
2) $f-c$ is reducible for at least $d^{2}$ values of $c \in \bar{K}$,

3) $f \in \bar{K}\left[p^{2}, p^{3}\right]$ for some $p \in \bar{K}[x]$,

4) $\operatorname{gcd}\left\{\frac{\partial}{\partial x_{1}} f, \frac{\partial}{\partial x_{2}} f, \ldots, \frac{\partial}{\partial x_{n}} f\right\} \notin \bar{K}^{*}$,

we have 1) $\Longrightarrow 2) \Longrightarrow 3$ ) $\Longrightarrow$ 4).

Proof. If $f \in \bar{K}$, then 2), 3) and 4) are trivially satisfied, so assume that $f \notin \bar{K}$.

3) $\Rightarrow 4$ ) Suppose that 3) holds. Then $0<\operatorname{deg} p<\operatorname{deg} f$. Since $\frac{\partial f}{\partial p} \notin \bar{K}$ and $\frac{\partial f}{\partial p} \mid \frac{\partial f}{\partial x_{i}}$ for all $i$, we obtain 4$)$.

$2) \Rightarrow 3$ ) Suppose that 2) holds. From Corollary 3 of [Sch, Th. 37], it follows that $f=g(p)$ for some $g \in \bar{K}[t]$ of degree $\geq 2$ and some $p \in \bar{K}[x]$. Let $c$ be a root of $g^{\prime}$. If we replace $g(t)$ by $g(t+c)$ and $p$ by $p-c$, then we still have $f=g(p)$, but $g^{\prime}(0)$ becomes 0 . Hence $g$ has no linear part any more, and 3 ) follows.

1) $\Rightarrow 2$ ) Suppose that we have a decomposition $f-g=h_{1} \cdot h_{2}$ over $\bar{K}$, where $\operatorname{deg} h_{i} \geq 1$ for both $i$. If $h_{1} \in \bar{K}[y]$, then the leading homogeneous part with respect to $x$ of $f-g$ is divisible by $h_{1}$. This contradicts that $f-g$ has no monomials with variables of both $x$ and $y$, so $\operatorname{deg}_{x} h_{1}>0$ and $\operatorname{deg}_{x} h_{2}<\operatorname{deg}_{x} f$.

Consequently, $\operatorname{deg}_{x} h_{2}<\operatorname{deg}(f-c)$ and similarly $\operatorname{deg}_{x} h_{1}<\operatorname{deg}(f-c)$. For every $c \in \bar{K}, g=c$ has a solution $a \in \bar{K}^{n}$. Now $\left.\left.h_{1}\right|_{y=a} \cdot h_{2}\right|_{y=a}$ is a decomposition of $f-c$, where $\left.\operatorname{deg} h_{i}\right|_{y=a} \geq 1$ for both $i$ because $\left.\operatorname{deg} h_{3-i}\right|_{y=a}<\operatorname{deg} f$ for both $i$.

In 3) of the lemma below, $i \leq j$ is written instead of $i<j$ to include the case $n=1$, where 2) and 3) of the lemma below are always satisfied (take $p=x_{1}$ and $q=1$ in 2)).

Lemma 4.6. Assume that $f, g \in \bar{K}[x]$ have degree at most $d$. Then for

1) $f-c g$ is reducible for at least $d^{2}$ values of $c \in \bar{K}$ and $\operatorname{gcd}\{f, g\} \in \bar{K}$,

2) there exist $p, q \in \bar{K}[x]$ such that $f \in \bar{K}[p, q]$ and $g \in \bar{K}\left[p^{2}, p q, q^{2}, p^{3}, p^{2} q\right.$, $\left.p q^{2}, q^{3}\right]$,

3) $\operatorname{gcd}\left\{\operatorname{det} \mathcal{J}_{x_{i}, x_{j}}(f, g) \mid 1 \leq i \leq j \leq n\right\} \notin \bar{K}^{*}$.

we have 1) $\Longrightarrow 2) \Longrightarrow 3$ ).

Proof. If $f g=0$, then 1) $\Longrightarrow 2$ ) follows, because $f, g \in \bar{K}$ if $f g=0$ and $\operatorname{gcd}\{f, g\} \in \bar{K}$. If $f g \neq 0$, then 1) $\Longrightarrow 2$ ) follows from Corollary 2 of [Sch, Th. 37], because $f+x_{n+1} g$ is irreducible if $\operatorname{gcd}\{f, g\} \in \bar{K}$ and $f g \neq 0$. So 1$) \Longrightarrow 2$ ) is satisfied in any case.

If $\mathcal{J} f$ and $\mathcal{J} g$ are linearly dependent as row vectors over $\bar{K}(x)$, then the formula in 3) equals zero, so assume the opposite. Then $f$ and $g$ are algebraically 
independent over $\bar{K}$. Suppose that 2) holds. Then $p$ and $q$ are algebraically independent over $\bar{K}$ as well, and by the chain rule

$$
\mathcal{J}_{x_{i}, x_{j}}(f, g)=\mathcal{J}_{p, q}(f, g) \cdot \mathcal{J}_{x_{i}, x_{j}}(p, q)
$$

Hence $\operatorname{det} \mathcal{J}_{p, q}(f, g)$ divides the formula in 3$)$. Since both entries of $\mathcal{J}_{p, q} f$ are contained in $\bar{K}[p, q]$, and both entries of $\mathcal{J}_{p, q} g$ are linear combinations over $\bar{K}$ of terms $p^{i} q^{j}$ with $i+j \geq 1$, we have $\operatorname{det} \mathcal{J}_{p, q}(f, g) \in \bar{K}[p, q] \backslash \bar{K}$, and 3) follows. This gives 2$) \Longrightarrow 3$ ).

\section{$5 \quad$ Irreducibility results for arbitrary Keller maps}

Theorem 5.1. Let $F \in K[x]^{n}$ be a Keller map of degree d. Take $i \in\{1,2, \ldots, n$, $n+1\}$ and fix $\mu_{1}, \ldots, \mu_{i-1}, \mu_{i+1}, \ldots, \mu_{n+1} \in \bar{K}$. If $\mu_{j} \neq 0$ for some $j$ with $i \neq j \leq n$, then

$$
f:=\mu_{1} F_{1}+\mu_{2} F_{2}+\cdots+\mu_{n} F_{n}+\mu_{n+1}
$$

is reducible over $\bar{K}$ for at most $d^{2}-1$ values of $\mu_{i} \in \bar{K}$.

Proof. Assume without loss of generality that $j=1$. Then $\left(f, F_{2}, \ldots, F_{n}\right)$ is a Keller map as well. By expansion of the Jacobian determinant along the first row, we see that $\operatorname{gcd}\left\{\frac{\partial}{\partial x_{1}} f, \frac{\partial}{\partial x_{2}} f, \ldots, \frac{\partial}{\partial x_{n}} f\right\} \in K^{*} \subseteq \bar{K}^{*}$. Hence the case $i=n+1$ follows from 2) $\Longrightarrow 4$ ) of Lemma 4.5.

If $i \leq n$, then expansion of the Jacobian determinant along the first and the $i$-th row gives $\operatorname{gcd}\left\{\operatorname{det} \mathcal{J}_{x_{k}, x_{l}}\left(f, F_{i}\right) \mid 1 \leq k<l \leq n\right\} \in K^{*} \subseteq \bar{K}^{*}$. So the case $i \leq n$ follows from 1) $\Longrightarrow 3$ ) of Lemma 4.6 .

Corollary 5.2. Assume that $F \in K[x]^{n}$ is a Keller map. Then there exists $a \lambda \in K^{n}$ and $a T \in \mathrm{GL}_{n}(K)$ such that the components of both $F+\lambda$ and $T^{-1} F(T x)$ are all irreducible over $\bar{K}$.

Proof. Notice that $\# K \geq d^{2}$ because $\operatorname{chr} K=0$, where $d:=\operatorname{deg} F$. The first claim follows from the case $i=n+1$ of Theorem 5.1 and the second claim follows from the case $i \leq n$ of Theorem 5.1 with $\mu_{n+1}=0$.

In [KS, Th. 3], the authors proved additional properties for the $T \in \mathrm{GL}_{n}(K)$ when $n \geq 3$ and $K \subseteq \mathbb{C}$, namely that there exists a $T \in \mathrm{GL}_{n}(K)$ such that for every $\lambda \in K^{n}$, every component of $T^{-1} F(T x)+\lambda$ is irreducible over $\bar{K}$.

Lemma 5.3. Assume $F \in K[x]^{n}$ is any polynomial map over $K$. Let $d \geq 2$ be an integer and $\lambda \in K^{n}$. Then for all $h \in K\left[x, x_{n+1}\right]$, the map $G=(F-$ $\left.\lambda x_{n+1}^{d}, x_{n+1}, x_{n+2}-h\right)$ has the property that for all $\mu \in K^{n+3}$, either

$$
g:=\mu_{1} G_{1}+\mu_{2} G_{2}+\cdots+\mu_{n+2} G_{n+2}+\mu_{n+3}
$$

is irreducible, or $\mu_{n+1}=\mu_{n+2}=0$ and

$$
f:=\mu_{1} F_{1}+\mu_{2} F_{2}+\cdots+\mu_{n} F_{n}+\mu_{n+3}=g,
$$

or we have $\mu_{n+2}=0$ and $f \in \bar{K}\left[p^{2}, p^{3}\right]$ for some $p \in \bar{K}[x]$, in which case $\mathcal{J} f$ is not unimodular. 
Proof. Assume that $h \in K\left[x, x_{n+1}\right]$ and that $g$ is reducible. Then $\mu_{n+2}=0$ because otherwise $g$ would be a tame coordinate. Hence $g-f \in K\left[x_{n+1}\right]$. Since the monomials of $g-f$ can only have degrees 1 and $d$, we even have $g-f \in K\left[x_{n+1}\right] \backslash K^{*}$. If $f=g$, then $\mu_{n+1}=0$ because $\mu_{n+1} x_{n+1}$ is the difference between the linear parts of $g$ and $f$. If $f \neq g$, then by 1) $\Longrightarrow 3$ ) of Lemma 4.5 (with $f-g$ instead of $g$ ), $f \in \bar{K}\left[p^{2}, p^{3}\right]$ for some $p \in \bar{K}[x]$, and 3) $\Longrightarrow 4$ ) of Lemma 4.5 tells us $\mathcal{J} f$ is not unimodular over $\bar{K}$ and hence neither over $K$.

Lemma 5.4. Assume that $F \in K[x]^{n}$ is any polynomial map over $K$. Let $\Lambda \in K[y]^{n}$ such that $y_{1}, y_{2}, \ldots, y_{n}, \Lambda_{1}, \Lambda_{2}, \ldots, \Lambda_{n}, 1$ are linearly independent over $K$. Then for all $H \in K[x, y]^{n}$, the map

$$
G:=(F-\Lambda, y, z-H)
$$

has the property that for all $\mu \in K^{3 n+1}$, either

$$
g:=\mu_{1} G_{1}+\mu_{2} G_{2}+\cdots+\mu_{3 n} G_{3 n}+\mu_{3 n+1}
$$

is irreducible, or $\mu_{2 n+1}=\mu_{2 n+2}=\cdots=\mu_{3 n}=0$ and for

$$
f:=\mu_{1} F_{1}+\mu_{2} F_{2}+\cdots+\mu_{n} F_{n}+\mu_{3 n+1},
$$

we have $f \in \bar{K}\left[p^{2}, p^{3}\right]$ for some $p \in \bar{K}[x]$, in which case $\mathcal{J} f$ is not unimodular. Proof. Assume that $H \in K[x, y]^{n}$ and suppose that $g$ is reducible. Then $\mu_{2 n+1}=\mu_{2 n+2}=\cdots=\mu_{3 n}=0$ because otherwise $g$ would be a tame coordinate. Hence $g-f \in K[y]$. Since 1 is linearly independent over $K$ of $y_{1}, y_{2}$, $\ldots, y_{n}, \Lambda_{1}, \Lambda_{2}, \ldots, \Lambda_{n}$, as opposed to $g-f$, we even have $g-f \in K[y] \backslash K^{*}$. If $f=g$, then the linear independence over $K$ of $y_{1}, y_{2}, \ldots, y_{n}, \Lambda_{1}, \Lambda_{2}, \ldots, \Lambda_{n}$ tells us that $f=\mu_{3 n+1} \in \bar{K}\left[p^{2}, p^{3}\right]$ for any $p \in \bar{K}[x]$ and that $\mathcal{J} f=\left(\begin{array}{llll}0^{1} & 0^{2} & \cdots & 0^{n}\end{array}\right)$ is not unimodular. The case $f \neq g$ follows in a similar manner as in the proof of Lemma 5.3.

Corollary 5.5. Assume that $F \in K[x]^{n}$ is a Keller map over $K$ and $d \geq 2$ be an integer. Then for all $H \in K[x, y]^{n}$, the map

$$
G:=\left(F-y^{* d}, y, z-H\right)
$$

has the property that

$$
\mu_{1} G_{1}+\mu_{2} G_{2}+\cdots+\mu_{3 n} G_{3 n}+\mu_{3 n+1}
$$

is irreducible for all $\mu \in K^{3 n+1}$ such that $\mu_{i} \neq 0$ for some $i \leq 3 n$.

Proof. Assume that $H \in K[x, y]^{n}$ and suppose that $g:=\mu_{1} G_{1}+\mu_{2} G_{2}+\cdots+$ $\mu_{3 n} G_{3 n}+\mu_{3 n+1}$ is reducible and $\mu_{i} \neq 0$ for some $i \leq 3 n$. Since the linear parts of the components of $G$ are linearly independent over $K$, we see that $g \neq 0$. Let $f:=\mu_{1} F_{1}+\mu_{2} F_{2}+\cdots+\mu_{n} F_{n}+\mu_{3 n+1}$. By Lemma 5.4, we have $i \leq 2 n$. Since $\operatorname{deg} g \neq 1$ by reducibility of $g$, we can even take $i \leq n$.

Again by Lemma $5.4, \mathcal{J} f$ is not unimodular, so $\left(F_{1}, \ldots, F_{i-1}, f, F_{i+1}, \ldots, F_{n}\right)$ is not a Keller map. This contradicts that $F$ is a Keller map, so $g$ is irreducible if $\mu_{i} \neq 0$ for some $i \leq 3 n$. 
The proposition below can be used to show that $F_{i}-c$ is irreducible for all $c \in K$ in some additional cases. If we take for $v$ the first column of $T^{-1}$, where $T$ is as in the proof of Proposition 3.4, then

$$
T_{2} v=0 \neq T_{1} v \quad \text { and } \quad \mathcal{J} h \cdot v=0 \neq \mathcal{J} f \cdot v
$$

where $h$ and $f$ are as in Proposition 3.4. So Proposition 5.6 generalizes Proposition 3.4 in some sense.

Proposition 5.6. Assume that $f, h \in K[x]$ such that $\operatorname{deg}(f-h)=1$. If there exists a vector $v \in K^{n}$ such that $\mathcal{J} h \cdot v=0 \neq \mathcal{J} f \cdot v$, then $f$ is a tame coordinate.

Proof. Since $v \neq 0$, there exists a $T \in \mathrm{GL}_{n}(K)$ such that $v=T e_{1}$, where $e_{1}$ is the first standard basis unit vector and hence $T e_{1}$ is the first column of $T$. Consequently,

$$
\mathcal{J}(h(T x)) \cdot e_{1}=\left.(\mathcal{J} h)\right|_{x=T x} \cdot v=0 \neq\left.(\mathcal{J} f)\right|_{x=T x} \cdot v=\mathcal{J}(f(T x)) \cdot e_{1} .
$$

It follows that $h(T x) \in K\left[x_{2}, x_{3}, \ldots, x_{n}\right]$ and $f(T x) \notin K\left[x_{2}, x_{3}, \ldots, x_{n}\right]$. Since $\operatorname{deg}(f(T x)-h(T x))=1$, we see that $f(T x)-h(T x)-c x_{1} \in K\left[x_{2}, x_{3}, \ldots, x_{n}\right]$ for some $c \in K^{*}$. Hence $f(T x)-c x_{1} \in K\left[x_{2}, x_{3}, \ldots, x_{n}\right]$ and $E:=\left(c^{-1} f(T x), x_{2}\right.$, $\left.x_{3}, \ldots, x_{n}\right)$ is an elementary invertible polynomial map. Since $f$ is the first component of $c E\left(T^{-1} x\right)$, we see that $f$ is a tame coordinate.

\section{Irreducibility results for cubic Keller maps with- out quadratic parts}

Theorem 6.1. Assume $F=x+H$ is a polynomial map over $K$, such that $H \in K[x]^{n}$ is cubic homogeneous and $\mathcal{J} H$ is nilpotent. Say that besides linear combinations of 1 only, there are exactly $s \geq 1$ linear combinations of $F_{1}, F_{2}, \ldots, F_{n}, 1$ which are reducible, if we do not count scalar multiples.

Then $s \leq n-4$ and there exists a $T \in \mathrm{GL}_{n}(K)$ such that the first $s$ components of $T^{-1} F(T x)$ are reducible. In particular, $s$ is finite and the first $s$ components of $T^{-1} F(T x)$ are the only linear combinations of the components of $T^{-1} F(T x)$ and 1 , which are reducible and not a linear combination of 1 only.

Proof. Notice that $F$ is a Keller map and therefore, $\mathcal{J} F_{i}$ is unimodular for each $i$. By 1) $\Longrightarrow 3$ ) of Corollary 4.4, all reducible linear combinations of $F_{1}, F_{2}, \ldots, F_{n}, 1$ are already linear combinations of $F_{1}, F_{2}, \ldots, F_{n}$. Replace $F$ by a linear conjugation of $F$ such that as many components of $F$ as possible become reducible, say that exactly $t$ such components become reducible. Assume without loss of generality that $F_{1}, F_{2}, \ldots, F_{t}$ are the reducible components of $F$.

It suffices to show that $t=s$ and

$$
t \leq n-4
$$

We first show (10) by distinguishing $t>n-4$ into three cases. 
- $t>n-4 \leq 0$.

Then $n \leq 4$ and we have $s=t=0$ on account of E. Hubbers' result that the JC holds for $F$, see [Hub] or [vdE, Cor. 7.1.3]. This contradicts $s \geq 1$.

- $t>n-4>0$ and for each $i \leq t$, there exists a $j \leq t$ such that $H_{j}=\lambda_{j} x_{i} x_{j}^{2}$ for some $\lambda_{j} \in K^{*}$.

Notice that $j$ as above is unique for all $i \leq t$, hence $i \mapsto j$ is a permutation of $\{1,2, \ldots, t\}$, say with a cycle of length $k \leq t$. Then we may assume without loss of generality that

$$
H_{1}=\lambda_{1} x_{k} x_{1}^{2}, \quad H_{2}=\lambda_{2} x_{1} x_{2}^{2}, \quad H_{3}=\lambda_{3} x_{2} x_{3}^{2}, \quad \ldots, \quad H_{k}=\lambda_{k} x_{k-1} x_{k}^{2} .
$$

The leading principal minor determinant of size $k$ of $\mathcal{J} H$ equals

$$
\left(2^{k}-(-1)^{k}\right) \lambda_{1} x_{1}^{2} \lambda_{2} x_{2}^{2} \cdots \lambda_{k} x_{k}^{2},
$$

so the corresponding submatrix is not nilpotent. But since $F_{i} \in K\left[x_{1}, x_{2}\right.$, $\left.\ldots, x_{k}\right]$ for all $i \leq k$, the leading principal minor matrix of size $k$ is nilpotent, because its $p$-th power is a submatrix of $(\mathcal{J} H)^{p}$. We obtain a contradiction.

- $t>n-4>0$ and for some $i \leq t$, there does not exist $a j \leq t$ such that $H_{j}=\lambda_{j} x_{i} x_{j}^{2}$ for some $\lambda_{j} \in K^{*}$.

Notice that $\left.(\mathcal{J} H)\right|_{x_{i}=0}$ is nilpotent because $\mathcal{J} H$ is nilpotent. Since $F_{i}=$ $x_{i}+H_{i}$ is reducible, it follows from 1$) \Longrightarrow 3$ ) of Corollary 4.4 that the $i$-th row of $\left.(\mathcal{J} H)\right|_{x_{i}=0}$ is zero. Hence the principal minor matrix that we obtain from $\left.(\mathcal{J} H)\right|_{x_{i}=0}$ by removing its $i$-th row and $i$-th column is nilpotent as well. This minor matrix is equal to

$$
\mathcal{J}_{x_{1}, \ldots, x_{i-1}, x_{i+1}, \ldots, x_{n}}\left(\left.H_{1}\right|_{x_{i}=0}, \ldots,\left.H_{i-1}\right|_{x_{i}=0},\left.H_{i+1}\right|_{x_{i}=0}, \ldots,\left.H_{n}\right|_{x_{i}=0}\right) .
$$

By 1$) \Longrightarrow 3$ ) of Corollary 4.4 we see that $x_{j}^{2} \mid H_{j}$ for each $j \leq t$. But by assumption on $i$, we have $x_{i} x_{j}^{2} \nmid H_{j}$ for each $j \leq t$. Hence by cubic homogeneity of $H_{j}$, we have $\left.H_{j}\right|_{x_{i}=0} \neq 0$ for each $j \leq t$ except $j=i$. So $\operatorname{deg} F_{j}=\left.\operatorname{deg} F_{j}\right|_{x_{i}=0}$ and $\operatorname{deg} g=\left.\operatorname{deg} g\right|_{x_{i}=0}$ for every $g \mid F_{j}$ and each $j \leq t$ except $j=i$.

As a consequence, $\left.F_{1}\right|_{x_{i}=0}, \ldots,\left.F_{i-1}\right|_{x_{i}=0},\left.F_{i+1}\right|_{x_{i}=0}, \ldots,\left.F_{t}\right|_{x_{i}=0}$ are all reducible. Make $\hat{F}$ from $\left.F\right|_{x_{i}=0}$ by removing the $i$-th component and substituting $x_{i+1}=x_{i}, x_{i+2}=x_{i+1}, \ldots, x_{n}=x_{n-1}$, in that order. Then $\hat{F}$ has $n-1$ components, of which the first $t-1$ are reducible. By induction on $n$, it follows that $(t-1) \leq(n-1)-4$, so $t \leq n-4$.

So it remains to show that $t=s$. Suppose therefore that $t \neq s$. Then $t<s$ and by maximality of $t$, we cannot get the first $t+1$ components of $F$ reducible by way of conjugation. Hence all linear combinations of components of $F$ which are reducible are already linear combinations of $F_{1}, F_{2}, \ldots, F_{t}$. Since $t<s$, there exists a linear combination of the form $\mu_{1} F_{1}+\mu_{2} F_{2}+\cdots+\mu_{t} F_{t}$ which is reducible, such that $\mu_{i} \neq 0$ for at least two $i$ 's. Hence we may assume that 
there is a reducible linear combination of the form $\mu_{1} F_{1}+\mu_{2} F_{2}+\cdots+\mu_{r} F_{r}$ with $\mu_{1} \mu_{2} \cdots \mu_{r} \neq 0$, where $2 \leq r \leq t$.

Assume first that there exist $i \leq r<k$ such that $\frac{\partial}{\partial x_{k}} H_{i} \neq 0$. On account of 1) $\Longrightarrow 3$ ) of Corollary 4.4, for each $j \leq r$, we have $H_{j}=x_{j}^{2} g_{j}$ for some linear form $g_{j}$. Therefore,

$$
\frac{\partial}{\partial x_{k}}\left(\mu_{1} H_{1}+\mu_{2} H_{2}+\cdots+\mu_{r} H_{r}\right)
$$

is a nontrivial $K$-linear combination of $x_{1}^{2}, x_{2}^{2}, \ldots, x_{r}^{2}$. Since the coefficient of $x_{1} x_{2}$ in $\left(\mu_{1} x_{1}+\mu_{2} x_{2}+\cdots+\mu_{r} x_{r}\right)^{2}$ is $2 \mu_{1} \mu_{2} \neq 0,\left(\mu_{1} x_{1}+\mu_{2} x_{2}+\cdots+\mu_{r} x_{r}\right)^{2}$ does not divide (11), and neither divides $\mu_{1} H_{1}+\mu_{2} H_{2}+\cdots+\mu_{r} H_{r}$. Now 1) $\Longrightarrow 3$ ) of Corollary 4.4 tells us that the Jacobian of $\mu_{1} F_{1}+\mu_{2} F_{2}+\cdots+\mu_{r} F_{r}$ is not unimodular. Hence $\left(\mu_{1} F_{1}+\mu_{2} F_{2}+\cdots+\mu_{r} F_{r}, F_{2}, F_{3}, \ldots, F_{n}\right)$ is not a Keller map and neither is $F$. This contradicts that $\mathcal{J} H$ is nilpotent.

Assume next that $\frac{\partial}{\partial x_{k}} H_{i}=0$ for all $i \leq r<k$. Then $H_{i} \in K\left[x_{1}, x_{2}, \ldots, x_{r}\right]$ for all $i \leq r$ and the leading principal minor matrix of size $r$ of $\mathcal{J} H$ is nilpotent because its $p$-th power is a submatrix of $(\mathcal{J} H)^{p}$. Hence the map $\tilde{F}$ which consists of the first $r$ components of $F$ satisfies the conditions on $F$ of this theorem with $n=r$. On account of (10), at most $r-4$ components of $\tilde{F}$ can be reducible. This contradicts that all components of $\tilde{F}$ are reducible.

Corollary 6.2. Assume that $F \in K[x]^{n}$ is a cubic Keller map over $K$ without quadratic part. Then there exists a $\lambda \in K^{n}$ such that for all $h \in K\left[x, x_{n+1}\right]$, the map

$$
G:=\left(F-\lambda x_{n+1}^{3}, x_{n+1}, x_{n+2}-h\right)
$$

has the property that

$$
\mu_{1} G_{1}+\mu_{2} G_{2}+\cdots+\mu_{n+2} G_{n+2}+\mu_{n+3}
$$

is irreducible for all $\mu \in K^{n+3}$ such that $\mu_{i} \neq 0$ for some $i \leq n+2$.

Proof. Take $T$ as in Theorem 6.1 and let $\lambda$ be the sum of the columns of $T$. Assume that $g:=\mu_{1} G_{1}+\mu_{2} G_{2}+\cdots+\mu_{n+2} G_{n+2}+\mu_{n+3}$ is reducible for some $\mu \in K^{n+3}$ such that $\mu_{i} \neq 0$ for some $i \leq n+2$. If $f:=\mu_{1} F_{1}+\mu_{2} F_{2}+\cdots+$ $\mu_{n} F_{n}+\mu_{n+3}=g$, then $f(T x)=g(T x)$ is reducible as well, and by Theorem 6.1, $\left(\mu_{1} \mu_{2} \cdots \mu_{n}\right)$ is $c$ times a row of $T^{-1}$ for some $c \in K^{*}$. By the choice of $\lambda$, we have $g=f-\left(\begin{array}{llll}\mu_{1} & \mu_{2} & \cdots & \mu_{n}\end{array}\right) \lambda x_{n+1}^{3}=f-c x_{n+1}^{3}$ in this case, which contradicts $f=g$.

So $f \neq g$ and by Lemma 5.3, $\mathcal{J} f$ is not unimodular and $\mu_{n+2}=0$. Since $\mu_{n+1} G_{n+1}=\mu_{n+1} x_{n+1}$ is irreducible if $\mu_{n+1} \neq 0$, we can choose $i \leq n$. Hence $\left(F_{1}, \ldots, F_{i-1}, f, F_{i+1}, \ldots, F_{n}\right)$ is a Keller map as well as $F$. This contradicts that $\mathcal{J} f$ is not unimodular, so $g$ is irreducible. 


\section{$7 \quad$ Irreducibility results for symmetric Keller maps}

Lemma 7.1. Assume $F$ is a Keller map in dimension $n$ over $K$, such that $\mathcal{J} F$ is symmetric. If $F_{i}$ is of the form $c^{\prime} x_{i}+x_{i}^{2} h+c$ for some $c, c^{\prime} \in K$ and some $h \in K[x]$, then $h=0$.

Proof. Suppose that $F_{i}$ is of the form $c^{\prime} x_{i}+x_{i}^{2} h+c$. Then the constant part of $\mathcal{J} F_{i}$ is of the form

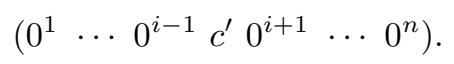

By expansion along the $i$-th row of $\mathcal{J} F$, we see that $p=\operatorname{det} \mathcal{J} F$ can be expressed as $p=p_{0}+(\mathcal{J} F)_{i i} p_{1}$, where both $p_{0}$ and $p_{1}$ are polynomials in the entries of $\mathcal{J} F$ except $(\mathcal{J} F)_{i i}$, in such a way that the constant part with respect to $x$ of $p_{0}$ is zero. We will use this to prove that $h=0$.

So let us assume that $h \neq 0$, say that $h=x_{i}^{r-2} \tilde{h}$, where $x_{i} \nmid \tilde{h}$. Then $F_{i}=c^{\prime} x_{i}+x_{i}^{r} \tilde{h}+c$. From Poincaré's lemma, it follows that $F=\nabla f$ for some $f \in K[x]$, so $\frac{\partial}{\partial x_{i}} f=F_{i}$. If $\tilde{h}$ has no monomials that are divisible by $x_{i}$, then

$$
f=\left.f\right|_{x_{i}=0} \cdot 1+c \cdot x_{i}+\frac{1}{2} c^{\prime} \cdot x_{i}^{2}+\frac{1}{r+1} \tilde{h} \cdot x_{i}^{r+1} .
$$

In the general case, we can turn out monomials of $\tilde{h}$ that are divisible by $x_{i}$ by reducing $\tilde{h} \cdot x_{i}^{r+1}$ modulo $x_{i}^{r+2}$, and we have

$$
f \bmod x_{i}^{r+2}=\left.f\right|_{x_{i}=0} \cdot 1+c \cdot x_{i}+\frac{1}{2} c^{\prime} \cdot x_{i}^{2}+\left.\frac{1}{r+1} \tilde{h}\right|_{x_{i}=0} \cdot x_{i}^{r+1} .
$$

where $\left.\tilde{h}\right|_{x_{i}=0} \neq 0$. Since $r \geq 2$ by definition, it follows that $(\mathcal{J} F)_{i i}=\frac{\partial^{2}}{\partial x_{i}^{2}} f$ is the only entry of the matrix $\mathcal{J} F=\mathcal{H} f$ with monomials of degree between 1 and $r-1$ inclusive in $x_{i}$, and those monomials add up to $\left.r \tilde{h}\right|_{x_{i}=0} \cdot x_{i}^{r-1} \neq 0$. Hence $p_{0}$ and $p_{1}$ do not have monomials of degree between 1 and $r-1$ inclusive in $x_{i}$ either. Furthermore, there exists a term $t$ of degree between 1 and $r-1$ inclusive in $x_{i}$ whose coefficient in $(\mathcal{J} F)_{i i}$ is nonzero. We can choose $t$ of minimum degree, so that $t$ is not divisible by any other such term of $(\mathcal{J} F)_{i i}$.

Since the coefficient of 1 in $p_{0}$ is zero, the coefficient of 1 in $p=\operatorname{det} \mathcal{J} F$, which is nonzero because of the Keller condition on $F$, is equal to the coefficient of 1 in $\left.(\mathcal{J} F)_{i i} \cdot p_{1}\right|_{x=0}$. The coefficient of $t$ in $\left.(\mathcal{J} F)_{i i} \cdot p_{1}\right|_{x=0}$ is nonzero as well, because $\left.p_{1}\right|_{x=0} \in K^{*}$ along with the coefficient of 1 in $\left.(\mathcal{J} F)_{i i} \cdot p_{1}\right|_{x=0}$.

So if we can show that the coefficient of $t$ in $p=\operatorname{det} \mathcal{J} F$ is equal to that in $\left.(\mathcal{J} F)_{i i} \cdot p_{1}\right|_{x=0}$, then we have a contradiction with the Keller condition on $F$, which gives us the conclusion that $h=0$. Indeed, since $p_{0}$ do not have monomials of degree between 1 and $r-1$ inclusive, the coefficient of $t$ in $p$ is equal to that in $(\mathcal{J} F)_{i i} \cdot p_{1}$. Now, $p_{1}$ does not have such monomials either, therefore our choice of $t$ tells us that coefficient of $t$ in $p$ is equal to that in $\left.(\mathcal{J} F)_{i i} \cdot p_{1}\right|_{x=0}$.

From now on in this section, we shall write $G_{i}^{(k)}$ for the homogeneous part of degree $k$ of a polynomial $G_{i}$ (which is the $i$-th component of a polynomial map 
$G$ ), or zero if $G_{i}$ has no such part. We did this already for $k=1$ in Theorem 3.3. Furthermore, we did something similar for the polynomials $f$ and $g$ in section 4.

Corollary 7.2. Assume $G$ is a Keller map in dimension $n$ over $K$, such that $\mathcal{J} G$ is symmetric. Suppose that $\frac{\partial}{\partial x_{i}} G_{i}^{(1)} \neq 0$.

If $G_{i}$ is of the form $G_{i}^{(1)}+\left(G_{i}^{(1)}\right)^{2} h+c$ for some $c \in K$ and $h \in K[x]$, then $h=0$ and hence $\operatorname{deg} G_{i}=1$.

Proof. Take $T \in \mathrm{GL}_{n}(K)$ such that $T$ corresponds to the identity matrix $I_{n}$ except for the $i$-th row, for which we take $\left(\frac{\partial}{\partial x_{1}} G_{i}^{(1)} \frac{\partial}{\partial x_{2}} G_{i}^{(1)} \ldots \frac{\partial}{\partial x_{n}} G_{i}^{(1)}\right)$. From Poincaré's lemma, it follows that $G=\nabla g$ for some $g \in K[x]$. Next, define $f:=g\left(T^{-1} x\right)$ and $F:=\left(T^{-1}\right)^{\mathrm{t}} G\left(T^{-1} x\right)$. Since $\left(T^{-1}\right)^{\mathrm{t}}$ corresponds to the identity matrix $I_{n}$ except for the $i$-th column, we have $F_{i}=\left(\left(T^{-1}\right)^{\mathrm{t}}\right)_{i} G\left(T^{-1} x\right)=$ $\left(T^{-1}\right)_{i i} G_{i}\left(T^{-1} x\right)$.

By definition of $T$, the linear part of $G_{i}$ is equal to $(T x)_{i}$, thus the linear part of $G_{i}\left(T^{-1} x\right)$ is equal to $G_{i}^{(1)}\left(T^{-1} x\right)=x_{i}$. Hence $F_{i}=\left(T^{-1}\right)_{i i} G_{i}\left(T^{-1} x\right)=$ $\left(T^{-1}\right)_{i i}\left(x_{i}+x_{i}^{2} h\left(T^{-1} x\right)+c\right)$. Furthermore,

$$
\nabla f=(\mathcal{J} f)^{\mathrm{t}}=\left(\mathcal{J} g\left(T^{-1} x\right)\right)^{\mathrm{t}}=\left(\left.G^{\mathrm{t}}\right|_{x=T^{-1} x} \cdot T^{-1}\right)^{\mathrm{t}}=\left(T^{-1}\right)^{\mathrm{t}} G\left(T^{-1} x\right)=F,
$$

so $\mathcal{J} F$ is symmetric, and we have $h\left(T^{-1} x\right)=0$ on account of Lemma 7.1. This gives the desired result.

Theorem 7.3. Assume $G$ is a Keller map in dimension $n$ over $K$, such that $\mathcal{J} G$ is symmetric. Suppose that $\frac{\partial}{\partial x_{i}} G_{i}^{(1)} \neq 0$ and $G_{i}^{(1)} \mid G_{i}^{(k)}$ for all $k \in\{1,2, \ldots$, $d-1\}$.

If $G_{i}-c$ has a divisor of degree less than $d$ with trivial constant part for some $c \in K$, then $\operatorname{deg} G_{i}=1$.

Proof. Suppose that $g \mid G_{i}-c, c \in K$, $\operatorname{deg} g<d$ and $g(0)=0$. Say that $G_{i}-c=g h$. Since $g(0)=0$, we have $h(0) \mid G_{i}^{(1)} \neq 0$. From Lemma 4.2 with $g^{*}=G_{i}^{(1)}$, we obtain that $G_{i}^{(1)}|g| G_{i}-c$. Hence we may assume that $g=G_{i}^{(1)}$.

By the Keller condition, $G_{i}$ is nonsingular over $\bar{K}$, which gives that $G_{i}$ is of the form of Corollary 7.2 above. On account of that corollary, $\operatorname{deg} G_{i}=1$.

Lemma 7.4. Let $f$ be of the form $g_{0}+g_{1} y_{1}+g_{2} y_{2}+\cdots+g_{n} y_{n} \neq g_{0}$, where $g_{i} \in A$ for all $i$ for some unique factorization domain $A$. Then $f$ is irreducible, if and only if $\operatorname{gcd}\left\{g_{0}, g_{1}, g_{2}, \ldots, g_{n}\right\} \in A^{*}$.

Proof. If $f$ decomposes in two factors, then one of the factors is constant with respect to $y$. Now the conclusion follows easily.

Lemma 7.5. Assume that $F \in K[x]^{n}$ is a polynomial map over $K$ and $f \in$ $K[x]$. Set $G:=\nabla_{x, y}\left(f+y^{\mathrm{t}} F\right)$. Then $\operatorname{det} \mathcal{J}_{x, y} G=(-1)^{n}(\operatorname{det} \mathcal{J} F)^{2}$ and $F$ is invertible, if and only if $G$ is invertible. Furthermore,

$$
\mu_{1} G_{1}+\mu_{2} G_{2}+\cdots+\mu_{n} G_{n}+\cdots+\mu_{2 n} G_{2 n}+\mu_{2 n+1}
$$


is irreducible for all $\mu \in K^{2 n+1}$ such that $\mu_{i} \neq 0$ for some $i \leq n$ if $F$ is a Keller map.

Proof. Notice that $\mathcal{J}_{x, y} G$ is of the form

$$
\mathcal{J}_{x, y} G=\mathcal{H}_{x, y}\left(f+y^{\mathrm{t}} F\right)=\left(\begin{array}{cc}
* & (\mathcal{J} F)^{\mathrm{t}} \\
(\mathcal{J} F) & 0
\end{array}\right)
$$

whence $\operatorname{det} \mathcal{J}_{x, y} G=(-1)^{n}(\operatorname{det} \mathcal{J} F)^{2}$. Consequently, both $F$ and $G$ are Keller maps if one of them is, which we assume from now on. Notice that $G=$ $\left((\mathcal{J} F)^{\mathrm{t}} y+\nabla f, F\right)$ by definition. Hence we have

$$
G\left(x,\left((\mathcal{J} F)^{\mathrm{t}}\right)^{-1}(y-\nabla f)\right)=(y, F),
$$

and we see that that $G$ is invertible, if and only if $F$ is invertible.

Suppose that $\mu \in K^{2 n+1}$ such that $\mu_{i} \neq 0$ for some $i \leq n$. Then (12) is of the form $g_{0}+g_{1} y_{1}+g_{2} y_{2}+\cdots+g_{n} y_{n}$ with $g_{i} \in K[x]$ for all $i$. More precisely,

$$
\left(g_{1} g_{2} \cdots g_{n}\right)=\mathcal{J}_{y}\left(\left(\mu_{1} \mu_{2} \cdots \mu_{n}\right)\left((\mathcal{J} F)^{\mathrm{t}} y+\nabla f\right)\right)
$$

is a nontrivial linear combination of the rows of $(\mathcal{J} F)^{\mathrm{t}}$, so $\left(g_{1} g_{2} \cdots g_{n}\right)$ is unimodular. In particular, $\operatorname{gcd}\left\{g_{0}, g_{1}, g_{2}, \ldots, g_{n}\right\} \in K^{*}$. Hence (12) is irreducible on account of Lemma 7.4.

Acknowledgment: The authors are very grateful to the referees who give some valuable advices.

\section{References}

[Bak1] Sławomir Bakalarski. Jacobian problem for factorial varieties. Univ. Iagel. Acta Math., (44):31-34, 2006.

[Bak2] Sławomir Bakalarski. The irreducibility of symmetric Yagzhev maps. Proc. Amer. Math. Soc., 138(7):2279-2281, 2010.

[BCW] Hyman Bass, Edwin H. Connell, and David Wright. The Jacobian conjecture: reduction of degree and formal expansion of the inverse. Bull. Amer. Math. Soc. (N.S.), 7(2):287-330, 1982.

[dBvdE1] Michiel de Bondt and Arno van den Essen. The Jacobian conjecture for symmetric Drużkowski mappings. Ann. Polon. Math., 86(1):43$46,2005$.

[dBvdE2] Michiel de Bondt and Arno van den Essen. A reduction of the Jacobian conjecture to the symmetric case. Proc. Amer. Math. Soc., 133(8):2201-2205 (electronic), 2005. 
[CvdE] Charles Ching-An Cheng and Arno van den Essen. Endomorphisms of the plane sending linear coordinates to coordinates. Proc. Amer. Math. Soc., 128(7):1911-1915, 2000.

[Dru1] Ludwik M. Drużkowski. An effective approach to Keller's Jacobian conjecture. Math. Ann., 264(3):303-313, 1983.

[Dru2] Ludwik M. Drużkowski. The Jacobian conjecture: symmetric reduction and solution in the symmetric cubic linear case. Ann. Polon. Math., 87:83-92, 2005.

[vdE] Arno van den Essen. Polynomial automorphisms and the Jacobian conjecture, volume 190 of Progress in Mathematics. Birkhäuser Verlag, Basel, 2000.

[vdES] Arno van den Essen and Vladimir Shpilrain. Some combinatorial questions about polynomial mappings. J. Pure Appl. Algebra, 119(1):47-52, 1997.

[Hub] E.-M.G.M. Hubbers. The Jacobian Conjecture: Cubic homogeneous maps in dimension four. Master's thesis, University of Nijmegen, Toernooiveld, 6525 ED Nijmegen, The Netherlands, February 171994. Directed by A.R.P. van den Essen.

[Jag] A. V. Jagžev. On a problem of O.-H. Keller. Sibirsk. Mat. Zh., 21(5):141-150, 191, 1980.

[Jag:E] A. V. Jagžev. Keller's problem. Siberian Math. J., 21(5):747-754, 1981. English translation of [Jag].

[Jẹd] Piotr Jẹdrzejewicz. A characterization of Keller maps. J. Pure Appl. Algebra, 217(1):165-171, 2013.

[Jel1] Zbigniew Jelonek. A solution of the problem of van den Essen and Shpilrain. J. Pure Appl. Algebra, 137(1):49-55, 1999.

[Jel2] Zbigniew Jelonek. A solution of the problem of van den Essen and Shpilrain II. J. Algebra, 358:8-15, 2012.

[Kal] Shulim Kaliman. On the Jacobian conjecture. Proc. Amer. Math. Soc., 117(1):45-51, 1993.

[Kel] Ott-Heinrich Keller. Ganze Cremona-Transformationen. Monatsh. Math. Phys., 47(1):299-306, 1939.

[KS] Tadeusz Krasiński and Stanisław Spodzieja. On the irreducibility of fibres of complex polynomial mappings. Univ. Iagel. Acta Math., (39):167-178, 2001. Effective methods in algebraic and analytic geometry, 2000 (Kraków). 
[LM] Jeffrey Lang and Samer Maslamani. Some results on the Jacobian conjecture in higher dimension. J. Pure Appl. Algebra, 94(3):327-330, 1994.

[MYZ] Alexander A. Mikhalev, Jie-Tai Yu, and Andrej A. Zolotykh. Images of coordinate polynomials. Algebra Colloq., 4(2):159-162, 1997.

[Sch] A. Schinzel. Polynomials with special regard to reducibility, volume 77 of Encyclopedia of Mathematics and its Applications. Cambridge University Press, Cambridge, 2000. With an appendix by Umberto Zannier.

[Wan] Stuart Sui Sheng Wang. A Jacobian criterion for separability. J. Algebra, 65(2):453-494, 1980. 